\title{
Analysis of Hydrogen Rich PG 1159 Central Stars of Planetary Nebulae
}

\section{S. Dreizler, K. Werner and U. Heber}

Institut für Astronomie und Astrophysik Universität Kiel; Institut für Astronomie und Astrophysik Universität Tübingen; Dr. Remeis Sternwarte

The PG 1159 stars represent the hottest stage of post-AGB evolution. Quantitative spectral analyses of most known PG 1159 stars have been carried out by us from optical, UV and EUV observations (see Dreizler et al 1995 for a review). It has been shown that these stars have atmospheres dominated by $\mathrm{C}$ and $\mathrm{He}$ with a significant admixture of $\mathrm{O}$. These abundances reveal the inter-shell matter of a former AGB star. The four stars, HS 2324+3944, NGC 7094, Abell 43, and Sh 2-68, define a small group of peculiar PG 1159 stars (termed hybrid PG 1159). Unlike all other PG 1159 stars hydrogen is detected in their spectra. Three of them are CSPNe. Our Non-LTE analyses (Dreizler et al 1995; Dreizler et al 1996) show that these stars have typical PG $1159 \mathrm{~T}_{\text {eff }}, \log \mathrm{g}$ as well as $\mathrm{C}$ and $\mathrm{He}$ abundances (Table 1). In contrast, the $\mathrm{O}$ abundance is lower than in PG 1159 stars. $\mathrm{N}$ is probably present but near the detection limit of the currently available spectra. Hybrid PG 1159 stars tend to have lower masses/luminosities than ordinary PG 1159 stars. A reduced massloss in their post-AGB evolution might be responsible for the incomplete removal of the $\mathrm{H}$ rich envelope. However, peeling of a post-AGB star alone can not produce the observed abundance pattern. In addition, mixing is required. A first evolution calculation with time dependent mixing of Iben \& MacDonald (1995) shows some qualitative agreement in the abundance pattern like the $\mathrm{C} / \mathrm{He}$ ratio it is, however, not able to explain the overall abundances.

Non-radial g-mode pulsations in HS 2324+3944 were detected recently by Silvotti (1996). A possible detection in Abell 43 was also reported by Ciardullo \& Bond (1996). Since standard theory of pulsations in PG 1159 stars requires a hydrogen free envelope this is a challenge for the understanding of the GW Vir instability.

Table 1: Results of NLTE analyses of hybrid PG 1159 stars

\begin{tabular}{lcccccc} 
Star & $\mathrm{T}_{\text {eff }}$ & $\log \mathrm{g}$ & $\mathrm{He} / \mathrm{H}$ & $\mathrm{C} / \mathrm{H}$ & $\mathrm{M} / \mathrm{M}_{\odot}$ & $\log \mathrm{L} / \mathrm{L}_{\odot}$ \\
\hline HS 2324+3944 & 130000 & 6.2 & 0.5 & 0.15 & 0.59 & 3.4 \\
NGC 7094 & 110000 & 5.7 & 0.3 & 0.05 & 0.59 & 3.6 \\
Abell 43 & 110000 & 5.7 & 0.3 & 0.05 & 0.59 & 3.6
\end{tabular}

\section{REFERENCES}

Ciardullo R., Bond H.E. 1996, AJ 111, 2332

Dreizler S., Werner K., Heber U. 1995, in Lecture Notes in Physics 443, p. 160

Dreizler S., Werner K., Heber U., Engels 1996, A\&A 309, 820

Iben I. Jr., MacDonald J. 1995, in Lecture Notes in Physics 443, 48

Silvotti R. 1996, A\&A 309, L23 